\title{
Synthesis by Sol-gel Route and Characterization of Ceria Doped Silica Coatings on Commercial Carbon Steel
}

\author{
M.A. Domínguez-Crespo, ${ }^{1, *}$ E. Ramírez-Meneses, ${ }^{1}$ A.M. Torres-Huerta, ${ }^{1}$ \\ A. García-Murillo, ${ }^{1}$ E.M. Arce-Estrada, ${ }^{2}$ O.G. Castillo-Hernández ${ }^{3}$ \\ ${ }^{1}$ Centro de Investigación en Ciencia Aplicada y Tecnología Avanzada (CICATA-IPN) Km. 14.5 \\ Carr. Tampico-Puerto Industrial, C.P. 89600, Altamira, Tamaulipas, México \\ ${ }^{2}$ Instituto Politécnico Nacional, Departamento de Metalurgia y Materiales. A.P. 75-874, 07300 \\ México, D.F. México \\ ${ }^{3}$ Estudiante del Programa de Posgrado en Tecnología Avanzada, \\ Unidad Altamira del IPN México
}

Received 25 April 2008; accepted 14 January 2009

\begin{abstract}
The use of silane formulations for the pre-treatment of metallic substrates prior to painting or as topcoat has been increasing during the recent years. The proposed formulations are very attractive because they present environmental friendliness, enhance adhesion properties and provide a barrier layer that delays the corrosion processes. One of the most used techniques to synthesized based- $\mathrm{SiO}_{2}$ coatings is solgel. The preparation of sol-gel coatings with specific chemical functions offers potential advantages over traditional methods as it offers tailoring of their structure, texture and thickness and allows the fabrication of large coatings. The present work investigates the electrochemical behaviour of commercial carbon steel coated with ceria doped $\mathrm{SiO}_{2}$ by sol-gel process in basic media. To evaluate the influence of temperature on adhesion and protective properties, the coatings were sintered at three different temperatures, 623 $\mathrm{K}, 723 \mathrm{~K}$ and $873 \mathrm{~K}$, for $3 \mathrm{~h}$. The anticorrosion behaviour, of the films has been characterized in an aggressive medium by electrochemical techniques, while structural characteristics and morphology was followed by X-Ray Diffraction, Fourier Transformed Infra-Red and Scanning Electron Microscopy techniques. The results indicated that the thin films act as a protective barrier against exposure to the corrosive medium ( $3.5 \mathrm{wt}-\% \mathrm{NaCl}$ solution) and increase the lifetime of the susbtrate.
\end{abstract}

Keywords: sol-gel process, $\mathrm{SiO}_{2}$ coatings, structural characterization, rare earths, corrosion prevention.

\footnotetext{
"Corresponding author. E-mail address: mdominguezc@ipn.mx
} 


\section{Introducción}

El acero al carbono es uno de los materiales estructurales con mayor aplicación industrial debido a su bajo costo, su alta conductividad térmica y resistencia mecánica. Sin embargo, es al mismo tiempo un material propenso a sufrir un deterioro importante debido a los procesos de corrosión y al desgaste en operación, afectando su tiempo de vida útil. Actualmente, diversas investigaciones se han dirigido a desarrollar recubrimientos económicos de baja toxicidad, que incrementen las propiedades de protección anticorrosiva, al desgaste y al mismo tiempo, que le confieran un acabado estético al material [13]. Dentro de éstas, se han explorado principalmente dos tendencias: 1) el uso de recubrimientos de protección activa, que forman nuevos compuestos por reacción con el sustrato y 2) los recubrimientos de auto-curado, que son capaces de retrasar el avance de la corrosión formando compuestos inertes por sí mismos. En sustratos metálicos, un aspecto importante para retrasar el avance de la corrosión es el papel de los inhibidores de corrosión, los cuales se adicionan en bajos porcentajes y son capaces de retrasar el fenómeno de corrosión sin causar algún cambio en las propiedades físicas. Las sales de lantánidos se han utilizado como pigmentos inhibidores de corrosión y se espera que sustituyan a los sistemas convencionales de protección. Los recubrimientos de conversión química y acabado final convencionales utilizan sales de cromo, fósforo y pinturas de óxido de plomo, entre otras; sin embargo, las regulaciones ambientales a nivel mundial han comenzado la prohibición de estas sustancias químicas, iniciándose la sustitución por sales de lantano. Por otro lado, el desarrollo de recubrimientos mediante la técnica de sol-gel es una de las alternativas importantes para incrementar el desempeño de estos materiales [4-7]. El proceso sol-gel es un método químico que permite la obtención de recubrimientos vítreos y cerámicos de alta densidad, a temperaturas relativamente bajas, a través de un procedimiento relativamente sencillo. Asimismo, mediante esta técnica se puede obtener la fabricación de materiales amorfos y policristalinos con características especiales en su composición y propiedades [1, 8-20]. Además, una de las ventajas más relevantes del método es que no requiere de altas temperaturas y condiciones especiales de vacío para llevar a cabo el proceso, como es el caso de los recubrimientos realizados por otras técnicas (CVD y PVD) [21, 22]. En este sentido, se ha reportado que las películas de $\mathrm{SiO}_{2}$ presentan excelentes propiedades de dureza, resistencia al desgaste, anticorrosivas, ópticas y dieléctricas, cuando se aplican en diversas clases de materiales [23-26]. Con el objeto de generar recubrimientos de baja toxicidad con características protectoras $\mathrm{y}$ resistentes al desgaste, en este trabajo se evaluaron las propiedades anticorrosivas de películas de $\mathrm{SiO}_{2}$ dopadas con $0.4 \%$ en peso de cerio, sintetizadas por la técnica de sol-gel en medio básico, utilizando como sustrato acero al carbono comercial (AISI-1012). 


\section{Experimental}

\section{Preparación de las muestras y recubrimientos}

Se utilizaron placas de acero al carbono AISI 1012 de 20 × 20 x $3 \mathrm{~mm}$ recubiertas con $\mathrm{SiO}_{2}$ dopado con $0.4 \%$ en peso de cerio, para pruebas de resistencia a la polarización y de 60 × 40 × $3 \mathrm{~mm}$, para pruebas de inmersión continua. Previo al proceso de recubrimiento, las muestras fueron desengrasadas, lijadas con carburo de silicio de 200, 400 y 600 micras para, posteriormente, desengrasarlas nuevamente con jabón, acetona y agua desionizada, de acuerdo con los estándares internacionales. La preparación del sol se llevó a cabo de la siguiente manera; se añadieron $7.5 \mathrm{~mL}$ de etanol por cada mililitro de TEOS $\mathrm{Si}\left(\mathrm{OC}_{2} \mathrm{H}_{5}\right)_{4}$ y se mezclaron mediante una agitación vigorosa durante una hora. Posteriormente, se agregó la disolución catalizadora $\left(0.038 \mathrm{M}\right.$ de $\left.\mathrm{NH}_{4} \mathrm{OH}\right)$ con una relación de $0.4 \mathrm{~mL}$ por cada $\mathrm{mL}$ de TEOS y agua en una relación 4:1 con respecto al mismo compuesto. Se procedió a una agitación durante un período de $12 \mathrm{~h}$, con el objeto de que se lleven a cabo las reacciones de hidrólisis y condensación, obteniendo así el sol de $\mathrm{SiO}_{2}$. El dopante de Ce se agregó al sol a partir de $\mathrm{Ce}\left(\mathrm{NO}_{3}\right)_{3} \cdot 6 \mathrm{H}_{2} \mathrm{O}$ para obtener un peso total de Ce de $0.4 \%$ y se agitó durante $1 \mathrm{~h}$.

La síntesis de las películas se realizó con una velocidad de $0.25 \mathrm{~cm} \mathrm{~s}^{-1}$ por la técnica de "dip-coating". Finalmente, se realizó un secado a $100{ }^{\circ} \mathrm{C}$ en una mufla durante 10 min, seguido de tratamientos térmicos a $300{ }^{\circ} \mathrm{C}, 400{ }^{\circ} \mathrm{C}$ ó $500{ }^{\circ} \mathrm{C}$ y posteriormente, las muestras se enfriaron a temperatura ambiente. Con el fin de incrementar el espesor de la película, se realizaron un total de 10 depósitos.

\section{Análisis de la microestructura y composición superficial de las películas}

La morfología de las muestras tratadas se analizó mediante microscopía electrónica de barrido acoplado con un detector de rayos-X (MEB/EDS), en un equipo JEOL JSM-6300. La presencia de grupos funcionales en el gel en muestras con tratamiento térmico $\left(300{ }^{\circ} \mathrm{C}, 400{ }^{\circ} \mathrm{C}\right.$ y $\left.500{ }^{\circ} \mathrm{C}\right)$ se analizaron mediante FT-IR (modelo Nicolet 5700) en un intervalo de frecuencia de 4000 $500 \mathrm{~cm}^{-1}$. Cada espectro se generó con un promedio de 5 ciclos a partir de una resolución nominal de $4 \mathrm{~cm}^{-1}$.

\section{Evaluación de las pruebas electroquímicas}

Pruebas electroquímicas tales como potencial a circuito abierto, polarización potenciodinámica (curvas de Tafel) y espectroscopia de impedancia electroquímica se realizaron utilizando una celda electroquímica convencional de 3 electrodos (referencia: electrodo de calomel saturado y contraelectrodo: grafito) en una disolución al $3.5 \%$ en peso de $\mathrm{NaCl}$ y temperatura ambiente, en un equipo AUTOLAB de la serie 30. Todas las pruebas se efectuaron después de una estabilización en el potencial de corrosión de $600 \mathrm{~s}$. Las medidas por la técnica de Tafel se realizaron con una velocidad de barrido de $2 \mathrm{mV} \mathrm{s}^{-1}$ desde 250 hasta $+250 \mathrm{mV}$. Las pruebas de espectroscopia de impedancia electroquímica (EIE) se realizaron en un intervalo de frecuencia de $100 \mathrm{KHz}$ a $0.01 \mathrm{~Hz}$, con una aplicación de la señal sinusoidal de potencial de $10 \mathrm{mV}$ a partir 
del potencial a circuito abierto. Cada experimento se repitió al menos 3 veces con el fin de presentar resultados reproducibles.

\section{Resultados y discusión}

La estructura y estabilidad térmica de las muestras evaluadas mediante análisis por espectroscopia de infrarrojo se muestran en la Fig. 1. En esta figura se pueden apreciar las diferentes señales que corresponden a las vibraciones de los grupos funcionales presentes en las películas sintetizadas a cada temperatura evaluada. Las bandas observadas en los intervalos de $430-500 \mathrm{~cm}^{-1}, 700-860 \mathrm{~cm}^{-1}$ y $1000-1100 \mathrm{~cm}^{-1}$ corresponden a las señales de flexión, estiramiento simétrico y asimétrico de $\mathrm{Si}-\mathrm{O}-\mathrm{Si}$, respectivamente. La señal observada en $900-980 \mathrm{~cm}^{-1}$ corresponde al estiramiento del enlace del grupo Si-OH. Las bandas observadas entre 3400 y $3700 \mathrm{~cm}^{-1}$ corresponden al grupo asimétrico $-\mathrm{OH}$, mientras que entre 1600-1700 $\mathrm{cm}^{-1}$ se observa la banda correspondiente a la deformación del $\mathrm{H}-\mathrm{O}-\mathrm{H}$. Estas últimas bandas corresponden a los grupos oxhidrilos y agua, que se encuentran presentes en los recubrimientos, por lo que su absorción disminuye con la temperatura de pre-tratamiento [27]. Los ensanchamientos ligeros observados en $2800-3000 \mathrm{~cm}^{-1}$ son atribuidos a las impurezas orgánicas que se incorporan al gel de silicio. En general, los espectros obtenidos en las películas con y sin la adición de cerio presentan comportamiento muy similar, debido probablemente a las bajas concentraciones de cerio utilizadas para el dopaje.

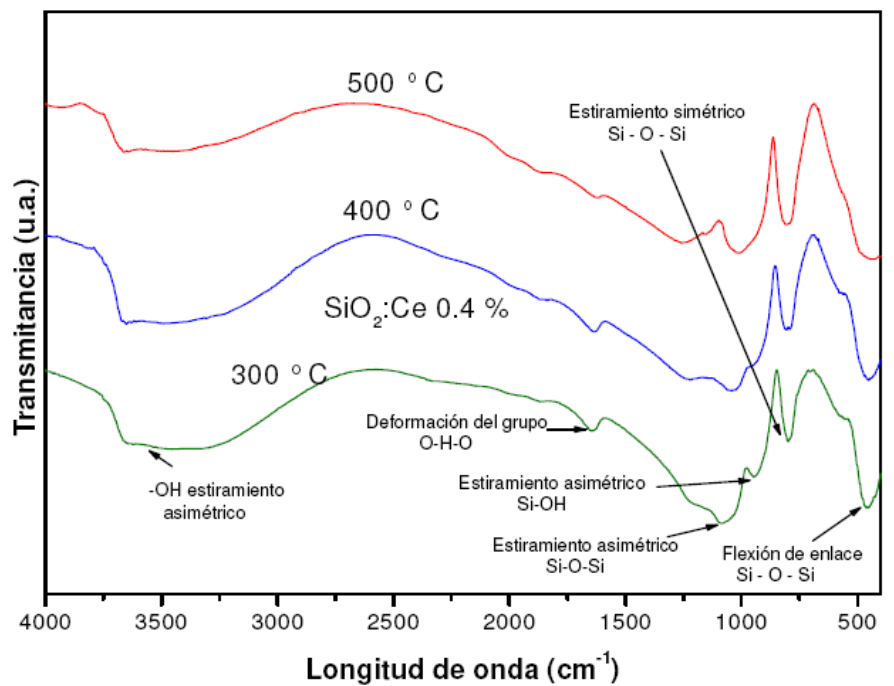

Figura 1. Diagramas FT-IR de las películas $\mathrm{SiO}_{2}$ dopadas con $0.4 \%$ en peso de cerio, después de tratarse térmicamente a 300,400 y $500{ }^{\circ} \mathrm{C}$.

La Fig. 2 muestra micrografías de MEB y sus correspondientes análisis de EDS de los recubrimientos de $\mathrm{SiO}_{2}$ :Ce antes de la evaluación electroquímica. A partir de estas imágenes puede observarse que las películas cubren uniformemente la superficie del substrato con una densidad de fracturas importante. Estas fracturas, o craqueos, son visiblemente afectados por la temperatura debido a que ocurre una densificación de la película. A la temperatura más baja de sinterización (300 
${ }^{\circ} \mathrm{C}$ ), el agrietamiento de la película es tan extenso que incluso parece que es poco adherente; sin embargo, con el incremento de la temperatura estos agrietamientos son cada vez menores, probablemente debido a la eliminación de los compuestos orgánicos.

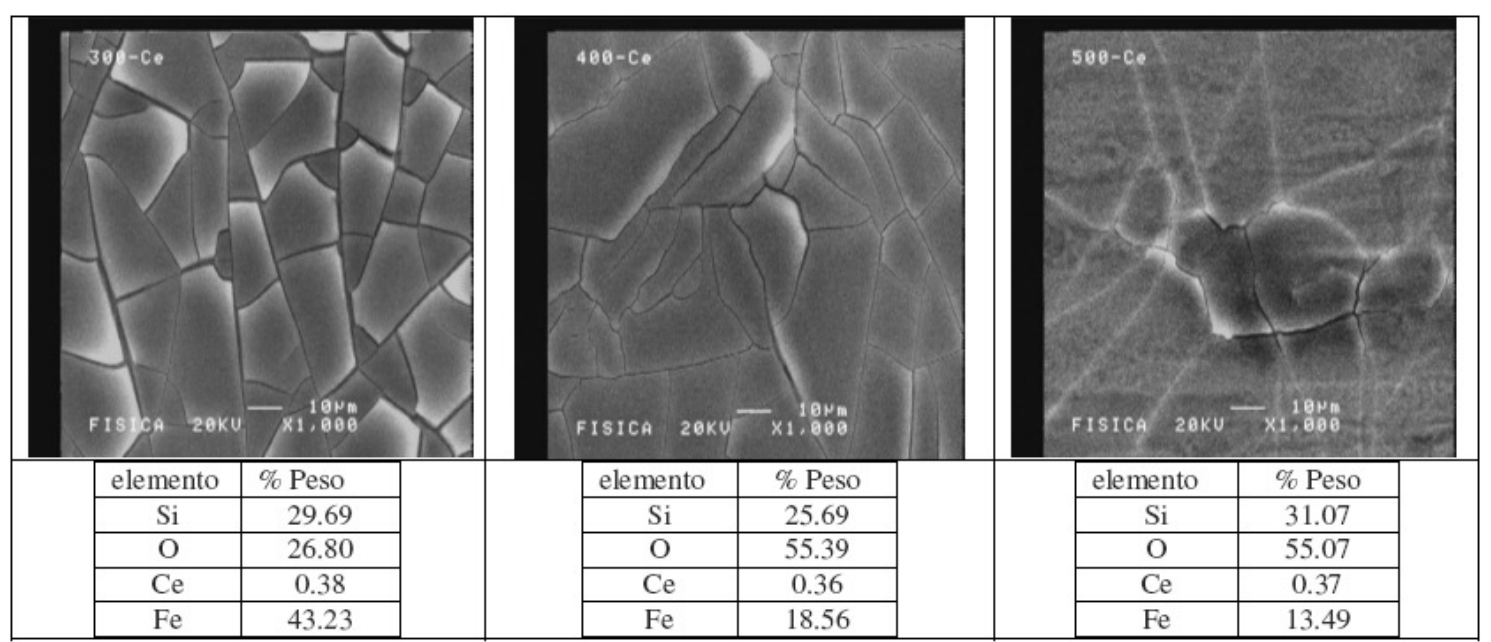

Figura 2. Micrografías obtenidas por MEB de las películas de $\mathrm{SiO}_{2}$ dopadas con $4 \% \mathrm{Ce}$ sobre substratos de acero a diferentes temperaturas: a) $300{ }^{\circ} \mathrm{C}$, b) $400{ }^{\circ} \mathrm{C} \mathrm{y} \mathrm{c)} 500{ }^{\circ} \mathrm{C}$.

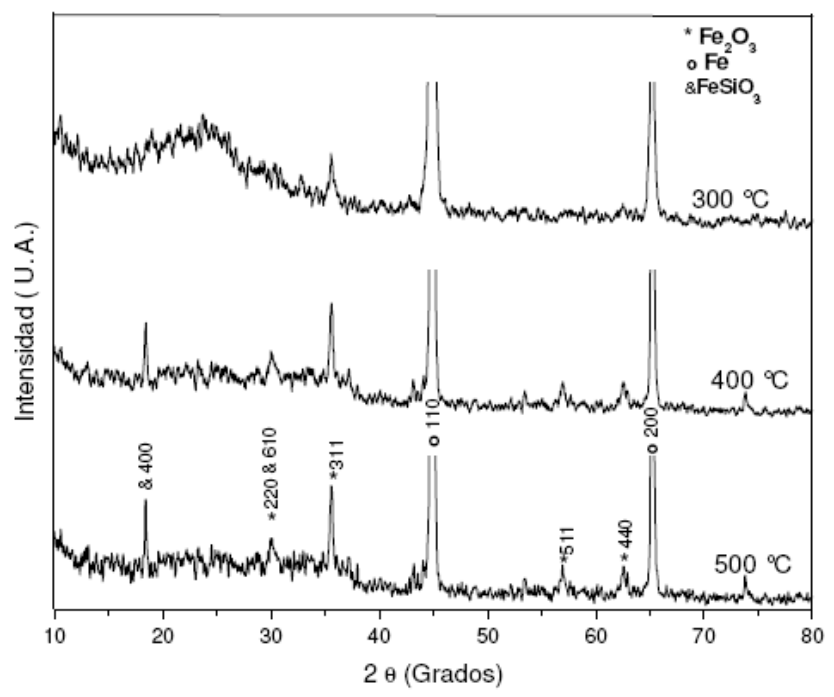

Figura 3. Espectros de difracción de rayos-X de las películas sinterizadas a diferentes temperaturas: a) $300{ }^{\circ} \mathrm{C}$, b) $400{ }^{\circ} \mathrm{C}$ y c) $500{ }^{\circ} \mathrm{C}$.

Los análisis de EDS muestran una importante cantidad de Fe que va disminuyendo con la temperatura de tratamiento, lo cual puede correlacionarse con las grietas observadas. Este craqueo puede explicarse si se consideran las diferencias en los coeficientes de expansión térmica entre el cerámico y el sustrato metálico, ocasionando que exista cierta difusión del Si hacia el substrato o bien, una difusión del hierro hacia la película, seguido de una reacción con el óxido de silicio, lo que resulta en la formación del compuesto $\mathrm{FeSiO}_{3}$, que fue identificado por análisis de difracción de rayos-X (Fig. 3). Por otro lado, se 
observa una ligera desviación de la cantidad nominal de Ce utilizada para dopar las películas de $\mathrm{SiO}_{2}$, lo cual puede atribuirse a varios factores, entre ellos, el que este tipo de análisis es únicamente cualitativo en una región pequeña de la muestra, o bien a la eficiencia obtenida durante el proceso sol-gel.

\section{Pruebas electroquímicas}

La Fig. 4 muestra las curvas potencial-tiempo de las películas de $\mathrm{SiO}_{2}$ con y sin la adición de cerio, además de la curva presentada para el acero desnudo (como referencia) en una disolución de $\mathrm{NaCl}$ al $3 \%$ en peso, a temperatura ambiente. En cada experimento, se realizaron al menos 5 medidas con el fin de determinar la reproducibilidad de las síntesis; sin embargo, en estas figuras solo se muestra la curva más representativa de cada una de ellas. En general, las evoluciones presentan dos regiones: i) durante los primeros tres minutos, una disminución relativamente rápida de potencial hacia valores más negativos y ii) una región donde el potencial disminuye muy lentamente hacia valores de equilibrio, hasta alcanzar un estado cuasi-estacionario. Como se puede observar en esta figura, el potencial de equilibrio es más positivo en las películas de $\mathrm{SiO}_{2}$ cuando se incrementa la temperatura; sin embargo, al adicionar el $\mathrm{Ce}$ se observan importantes cambios en cada uno de los tratamientos térmicos efectuados. A 300 y $500{ }^{\circ} \mathrm{C}$ puede apreciarse un desplazamiento negativo, mientras que a $400{ }^{\circ} \mathrm{C}$ el desplazamiento del potencial es anódico (positivo). La diferencia entre los potenciales se incrementa con la temperatura de sinterización. A partir de estos resultados, se determinó utilizar un potencial de estabilización de 10 minutos antes de realizar la evaluación mediante las técnicas de Tafel y EIE.

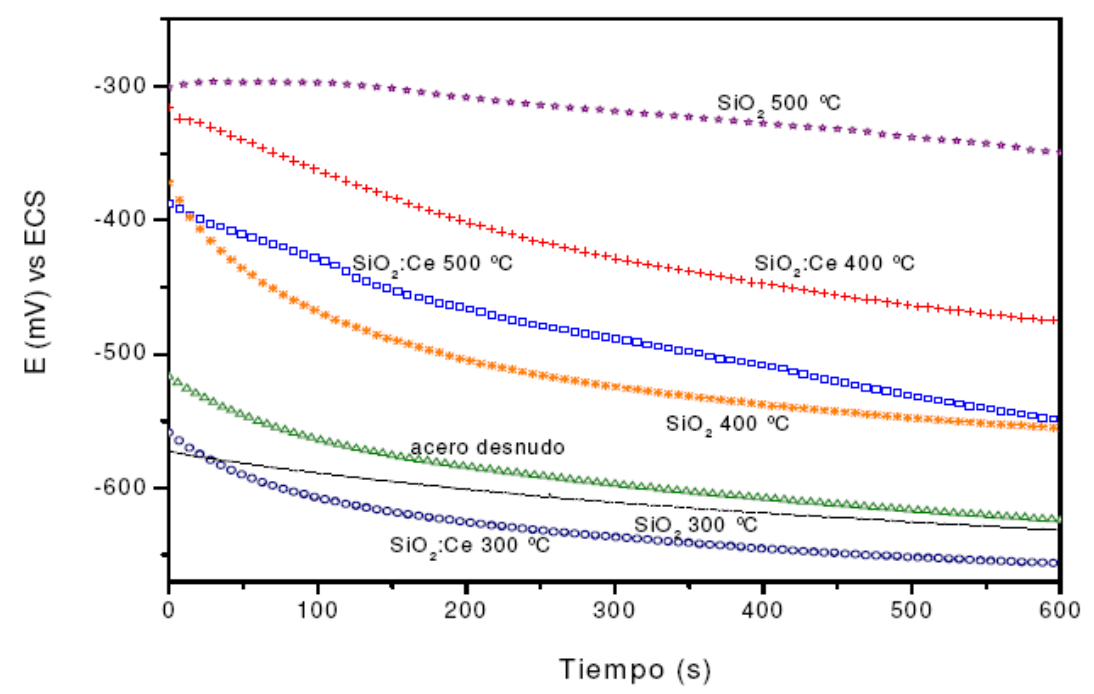

Figura 4. Curvas de potencial a circuito abierto, en donde se muestra la influencia de adicionar cerio en las películas de $\mathrm{SiO}_{2}$, con diferentes temperaturas de tratamiento. 


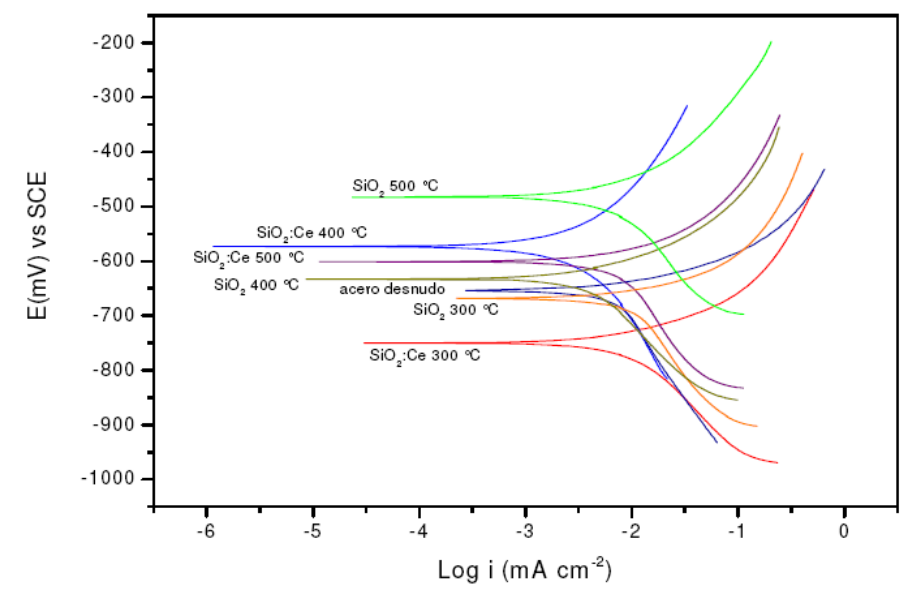

Figura 5. Curvas de Tafel obtenidas de los recubrimientos de $\mathrm{SiO}_{2}$ y $\mathrm{SiO}_{2}: \mathrm{Ce}$ sobre sustratos de acero comercial 1012.

Las curvas de Tafel de los recubrimientos obtenidos por el proceso sol-gel y evaluados en una disolución al $3 \%$ en peso, se presentan en la Fig. 5. Como se puede apreciar en esta figura, las velocidades de corrosión entre los diferentes recubrimientos (con y sin dopaje) son muy similares; sin embargo, comparando el recubrimiento con la velocidad de corrosión más baja $\left(\mathrm{SiO}_{2}: \mathrm{Ce}\left(400{ }^{\circ} \mathrm{C}\right)\right)$ y el acero desnudo, se obtiene un incremento en las propiedades de protección anticorrosiva de hasta un orden de magnitud (Tabla 1). Asimismo, se puede observar un desplazamiento en el potencial de corrosión con la temperatura de tratamiento térmico. En general el $\mathrm{E}_{\text {corr }}$ es más positivo cuando se incrementa la temperatura de tratamiento. Los desplazamientos observados en estas curvas tienen la misma tendencia que los analizados en las pruebas de potencial a circuito abierto. Sin embargo, es necesario mencionar que aún cuando la menor densidad de corriente se obtiene con los recubrimientos de $\mathrm{SiO}_{2}: \mathrm{Ce}$ tratados térmicamente a $400{ }^{\circ} \mathrm{C}$, estas tendencias representan un promedio sobre la superficie de las muestras y la densidad de corriente local que no fue calculada y, en consecuencia, el área real activa (la cual se incrementa con las micro-fisuras observadas en las micrografías de MEB) es desconocida. Sin embargo, se consideró que con los resultados de esta experimentación se puede obtener una buena aproximación.

Tabla 1. Densidades de corriente y potenciales de corrosión.

\begin{tabular}{ccc}
\hline Muestra & $\mathbf{i}_{\text {corr }}\left(\mathbf{A ~ c m} \mathbf{~ c m}^{-2}\right)$ & $\mathbf{E}_{\text {corr }}(\mathbf{m V})$ \\
\hline Acero desnudo & $2.9 \cdot 10^{-5}$ & -663 \\
Sustrato+ $\mathrm{SiO}_{2}: \mathrm{Ce}\left(300{ }^{\circ} \mathrm{C}\right)$ & $1.3 \cdot 10^{-5}$ & -779 \\
Sustrato+ $\mathrm{SiO}_{2}: \mathrm{Ce}\left(400^{\circ} \mathrm{C}\right)$ & $2.6 \cdot 10^{-6}$ & -576 \\
Sustrato+ $\mathrm{SiO}_{2}: \mathrm{Ce}\left(500{ }^{\circ} \mathrm{C}\right)$ & $9.8 \cdot 10^{-6}$ & -620 \\
Sustrato+ $\mathrm{SiO}_{2}\left(300{ }^{\circ} \mathrm{C}\right)$ & $2.8 \cdot 10^{-6}$ & -695 \\
Sustrato+ $\mathrm{SiO}_{2}\left(400{ }^{\circ} \mathrm{C}\right)$ & $9.6 \cdot 10^{-6}$ & -638 \\
Sustrato+ $\mathrm{SiO}_{2}\left(500{ }^{\circ} \mathrm{C}\right)$ & $8.7 \cdot 10^{-6}$ & -506 \\
\hline
\end{tabular}


Las propiedades de protección anticorrosiva de los recubrimientos con la adición de cerio se estudiaron mediante EIE y los resultados se presentan en la Fig. 6 a-c. En general, en el intervalo de frecuencias evaluado, los diagramas de Nyquist muestran la formación de un semicírculo deformado; esta deformación es regularmente asociada a las desviaciones del comportamiento ideal del recubrimiento; también, se observa la influencia de la temperatura de tratamiento y el dopaje con cerio en las películas sintetizadas (Fig. 6a). Particularmente, los recubrimientos sinterizados de $\mathrm{SiO}_{2}: \mathrm{Ce}$ a $400{ }^{\circ} \mathrm{C}$ presentaron el mayor incremento en las propiedades de protección anticorrosiva. Los diagramas de Bode en esta figura, confirman la presencia de un semicírculo a frecuencias altas e intermedias; sin embargo, para casi todas las muestras se observa una insinuación a la formación de una segunda constante de tiempo a frecuencias menores de $10^{-1} \mathrm{~Hz}$. Esta segunda señal podría relacionarse con la resistencia a la transferencia de carga del sustrato (Fig. 6b y 6c). El circuito equivalente propuesto a partir de los resultados obtenidos, se muestra en la Figura 6d y los resultados del ajuste, en la Tabla 2. En este circuito equivalente, Rs es la resistencia de la disolución, $\mathrm{R}_{\text {coat }}$ es la resistencia del recubrimiento, $\mathrm{CPE}_{\text {coat }}$ está relacionada con la capacitancia del recubrimiento, $R_{c t}$ es la resistencia a la transferencia de carga y $\mathrm{CPE}_{\mathrm{dl}}$ es la capacitancia de la doble capa electroquímica. $\mathrm{CPE}_{\text {coat }}$ se atribuye al comportamiento no ideal del recubrimiento debido a los defectos (fracturas o grietas), mientras que $\mathrm{CPE}_{\mathrm{dl}}$ se relaciona con la superficie involucrada en el proceso de corrosión del sustrato. Los valores de ajuste calculados confirman los resultados obtenidos mediante la técnica de Tafel e indican la influencia positiva que se puede alcanzar con el dopaje a temperaturas menores de $400{ }^{\circ} \mathrm{C}$. Las diferencias observadas en la resistencia de la disolución, pueden atribuirse a una pequeña disolución de los óxidos formados sobre la superficie de la película.

Tabla 2. Valores obtenidos para las películas de sol-gel, utilizando el circuito equivalente propuesto.

\begin{tabular}{|c|c|c|c|c|c|c|c|}
\hline \multirow[t]{2}{*}{ Muestra } & \multirow[t]{2}{*}{$\mathbf{R}_{\mathrm{s}}\left(\Omega \mathrm{cm}^{2}\right)$} & \multicolumn{2}{|c|}{$\mathrm{CPE}_{\text {coat }}$} & \multirow{2}{*}{$\begin{array}{c}\mathbf{R}_{\text {coat }} \\
\left(\Omega \mathrm{cm}^{2}\right)\end{array}$} & \multicolumn{2}{|c|}{$\mathrm{CPE}_{\mathrm{dl}}$} & \multirow[t]{2}{*}{$\mathbf{R}_{\mathrm{ct}}\left(\Omega \mathrm{cm}^{2}\right)$} \\
\hline & & $Y_{0} / \mathbf{F ~ ~ c m ^ { - 2 }}$ & $\mathbf{n}$ & & $Y_{0} / \mathbf{F ~ ~ c m ^ { - 2 }}$ & $\mathbf{n}$ & \\
\hline Bare steel & 23 & & - & - & $0.164 \cdot 10^{-5}$ & 0.76 & 993 \\
\hline $\mathrm{SiO}_{2}+\mathrm{Ce} 0.4 \%\left(300^{\circ} \mathrm{C}\right)$ & 30 & $0,910 \cdot 10^{-4}$ & 0.61 & 1739 & $0.812 \cdot 10^{-4}$ & 0.62 & 98 \\
\hline $\mathrm{SiO}_{2}+\mathrm{Ce} 0.4 \%\left(400^{\circ} \mathrm{C}\right)$ & 38 & $0.194 \cdot 10^{-4}$ & 0.81 & 16796 & $0.166 \cdot 10^{-4}$ & 0.77 & 52 \\
\hline $\mathrm{SiO}_{2}+\mathrm{Ce} 0.4 \%\left(500^{\circ} \mathrm{C}\right)$ & 22 & $0.229 \cdot 10^{-4}$ & 0.79 & 2901 & $0.680 \cdot 10^{-4}$ & 0.80 & 21 \\
\hline
\end{tabular}

Finalmente, los resultados obtenidos sugieren que las películas de $\mathrm{SiO}_{2}$ incrementan las propiedades de protección anticorrosiva al incrementar la temperatura y cuando se adiciona Cerio, se logra un beneficio hasta un límite que está relacionado con la porosidad y micro-fisuras observadas durante las medidas de MEB. 

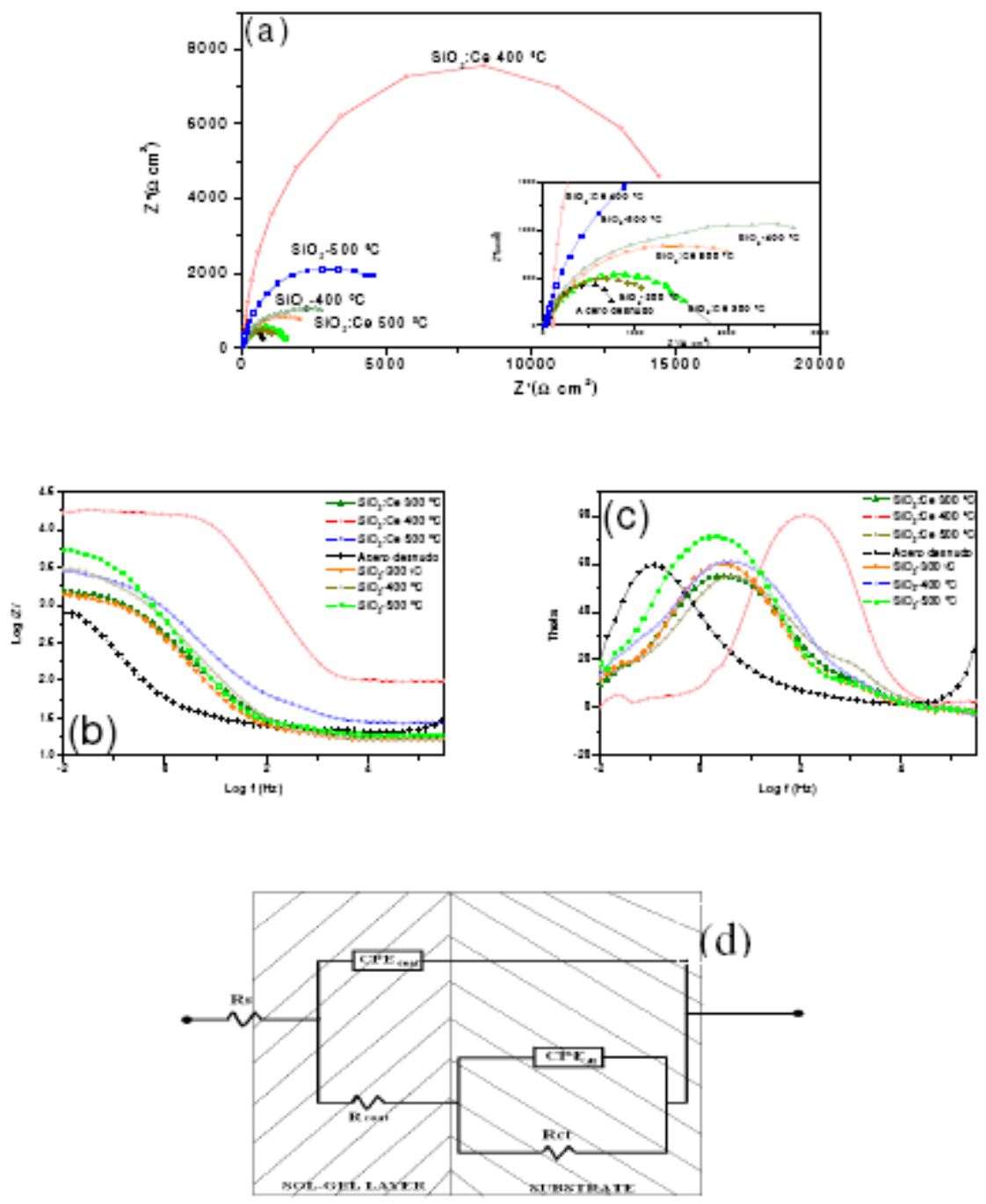

Figura 6. Diagramas de impedancia electroquímica de los recubrimientos evaluados en una disolución al $3.5 \%$ en peso de $\mathrm{NaCl}$ (a) Nyquist, (b) Modulo de impedancia, (c) ángulo de fase y (d) circuito equivalente propuesto.

\section{Conclusiones}

A partir de los resultados obtenidos en este trabajo, se pueden mencionar las siguientes conclusiones:

a) La temperatura de tratamiento influye fuertemente en la textura superficial y la capacidad anticorrosiva de estos recubrimientos. Sin embargo, este comportamiento puede ser modificado por la adición de una especie dopante en el sistema.

b) La presencia de $\mathrm{Ce}$ en la matriz de $\mathrm{SiO}_{2}$ y sinterizado a $400{ }^{\circ} \mathrm{C}$ aumenta la resistencia a la polarización hasta un factor $10^{4,7}$ y la velocidad de corrosión de estos recubrimientos es de un orden de magnitud comparable a la del acero desnudo.

c) El recubrimiento a $300{ }^{\circ} \mathrm{C}$ que exhibe menor protección, puede atribuirse en cierta medida a las fisuras observadas en las micrografías de MEB. Esto fragiliza el recubrimiento y puede dejar al descubierto el substrato provocándole menor protección. A partir de los análisis de morfología no es 
posible explicar el comportamiento electroquímico del recubrimiento obtenido a $400{ }^{\circ} \mathrm{C}$, debido a que todos los recubrimientos presentan fracturas en mayor o menor proporción, por lo que estudios más detallados se están realizando por este grupo de investigación.

\title{
Agradecimientos
}

El trabajo fue financiado por CONACYT, mediante los proyectos SEP-CONACyT 2006-01-61354, SIP 20080805, 20080654, 20082247 y 20080838.

\author{
Síntesis y caracterización electroquímica de películas de $\mathrm{SiO}_{2}$ dopadas con Cerio por la \\ técnica de sol-gel en medio básico
}

\begin{abstract}
Resumen
La síntesis de recubrimientos con base sílice, en el pretratamiento de diversos materiales antes de un acabado estético o final, se ha incrementado de manera importante en los últimos años. Las ventajas de este tipo de películas son su baja o nula toxicidad, incremento en las propiedades de adherencia y la generación de una barrera de protección capaz de retardar de manera considerable el proceso de corrosión. Una de las técnicas más utilizadas para la síntesis de estos recubrimientos es la técnica sol-gel, mediante la cual se pueden sintetizar películas con características específicas en estructura, textura, composición química y espesor en una amplia variedad de substratos. En este trabajo se presenta el comportamiento electroquímico de recubrimientos de $\mathrm{SiO}_{2}$ dopados con $0.4 \%$ en peso de cerio sobre acero al carbono comercial (1012), las cuales se sintetizaron por la técnica de sol-gel a partir de TEOS $\left(\mathrm{Si}\left(\mathrm{OC}_{2} \mathrm{H}_{5}\right)_{4}\right)$ en medio alcalino. Las muestras fueron tratadas térmicamente a tres temperaturas: 623, 723 y 873 $\mathrm{K}$ durante $3 \mathrm{~h}$, con el fin de evaluar la influencia en las propiedades de protección y adherencia. La evaluación electroquímica de las películas se realizó mediante las técnicas de Tafel y Espectroscopia de Impedancia Electroquímica, mientras que la evaluación estructural y morfológica se realizó mediante Difracción de rayos-X (DRX), Infrarrojo y Microscopía Electrónica de Barrido (MEB). Los resultados obtenidos indican que este tipo de recubrimientos actúa como una barrera de protección contra la corrosión en medios agresivos ( $\mathrm{NaCl} 3.5 \%$ en peso) incrementando el tiempo de vida media de esta clase de substratos.
\end{abstract}

Palabras Clave: sol-gel, recubrimientos base-sílice, caracterización estructural, tierras raras, prevención de la corrosión.

\section{Referencias}

1. S.J. Bull, R. Kingswell, K.T. Scott, Surf. Coat. Technol. 82 (1996) 218-225.

2. M. Saxena, B.K. Prasad, T.K. Dan, Plating and Surf. Finish. (1992) 57-61.

3. K.K. Biswas, S. Dutta, S.K. Das, M.C. Ghose, A. Mazumdar, N. Roy, Proc. Advances in Surface Treatment of Metals, ASTOM-87, BARC, Bombay (1987) 339.

4. M.G. Wankhede, A.B. Gaikwad, P.P. Patil, Surf. Coat. Technol. In press (2006).

5. H. Li, K. Liang, L. Mei, S. Gu, S. Wang, Mat. Lett. 51 (2001) 320-324.

6. M. Fallet, H. Mahdjoub, B. Gautier, J.P. Bauer, J. Non-Cryst. Solids 293-29 (2001) 527-533. 
7. M. Zhou, Q. Yang, T. Troczynski, Surf. Coat. Technol. 200 (2006) 28002804.

8. J.F. Quinson, C. Chino, M.D. Becdelievre, C. Guizard, M. Brunel, J. Mat. Sci. 31 (1996) 5179-5184.

9. T. Hubert, S. Svoboda, B. Oertel, Surf. Coat. Technol. 201 (1-2) (2006) 487-491.

10. M. Atik, C.R. Kha, P. Lima Neto, L.A. Avaca, M.A. Aegerter, J. Zarz, J. Mat. Sci. Lett. 14 (1995) 178-181.

11. M. Shame, M.L. Mecartnay, J. Mat. Sci. 25 (1990) 1537-1544.

12. A. Lgamiri, A. Guembour, A. Ben Bachir, S. EI Hajjaji, L. Aries, Surf. Coat.Technol. 162 (2003) 154-160.

13. S. Wilson, H.M. Howthorne, Q. Yang, T. Troczynski, Wear 251 (2001) 1042-1050.

14. S. Wilson, H.M. Howthorne, Q. Yang, T. Troczynski, Surf. Coat. Technol. 133-134 (2000) 389-396.

15. A. Balamurugan, S. Kannan, S. Rajeswari, Mat. Lett. 57 (2003) 4202-4205.

16. M. Sheffer, A. Groysman, D. Starosvetsky, N. Savchenko, D. Mandler, Corr. Sci. 46 (2004) 2975-2985.

17. C. Wang, F. Jiang, F. Wang, Corr. Sci. 46 (2004) 78-89.

18. A. Pepe, M. Aparicio, S. Cere, A. Duran, J. Non-Cryst. Solids 348 (2004) 162-171.

19. M.L. Zheludkevich, R. Serra, M.F. Montemor, KA. Yasakau, I.M. Miranda Salvado, M.G.S. Ferreira, Electrochim. Acta 51 (2005) 208-217.

20. A.L.K. Tan, A.M. Soutar, I.F. Annergren, Y.N. Liu, Surf. Coat. Technol. 198 (2005) 478-482.

21. K. Ortner, T. Jung, C.-P. Klages, B. Linder, B. Straussb, N. Samann, Surf. Coat. Technol. 200 (2005) 976-979.

22. T. Goto, Surface \& Coatings Technology 198 (2005) 367- 371

23. W.Q. Zhang, Y.X. Tang, Proc. SPIE Int. Soc. Opt. Eng. 3175 (1997) 94.

24. A.S. Hamdy, D.P. Butt , A.A. Ismail, Electrochim. Acta 52 (2007) 33103316.

25. M. Garcia-Heras, A. Jimenez-Morales, B. Casal, J.C. Galvan, S. Radzki, M.A. Villegas, J. Alloys \& Compounds 380 (2004) 219-224

26. B.D. Fabes, G.D. Berry, J. Non-Cryst. Solids 121 (1990) 357-364.

27. X. Zhang, Y. Wu, S. He, D. Yang, Surf. Coat. Technol. 201 (2007) 60516058. 